\title{
Patient perspectives on interprofessional collaboration between healthcare professionals during hospitalization: a qualitative systematic review protocol
}

\author{
Amélia Didier ${ }^{1,2,3}$ • Joan Campbell ${ }^{1,2} \cdot$ Loris Franco $^{2} \cdot$ Magali Serex $^{2} \cdot$ Liliana Staffoni-Donadini $^{2}$. \\ David Gachoud ${ }^{4}$. Maya Zumstein-Shaha ${ }^{1,3}$ \\ ${ }^{1}$ Bureau d'Echange des Savoirs pour des praTiques exemplaires de soins (BEST): a Joanna Briggs Institute Centre of Excellence, Lausanne, \\ Switzerland, ${ }^{2}$ School of Health Care Sciences (HESAV), University of Applied Sciences and Arts of Western Switzerland (HES-SO), Switzerland, \\ ${ }^{3}$ University Institute of Higher Education and Research in Health Care (IUFRS), University of Lausanne (UNIL), Lausanne, Switzerland, and \\ ${ }^{4}$ Department of Internal Medicine, University Hospital Lausanne (CHUV) and Medical Education Unit, Faculty of Biology and Medicine (FBM), \\ University of Lausanne (UNIL), Lausanne, Switzerland
}

Review question/objective: The objective of this qualitative systematic review is to examine the available evidence on interprofessional collaboration from the patients' perspectives, specifically on: i) interprofessional collaboration in adult or pediatric wards during hospitalization; ii) the influence of interprofessional collaboration on the patient's care, safety and well-being in adult or pediatric wards during hospitalization; and (iii) the patient's role in the interprofessional collaboration process in adult or pediatric wards during hospitalization.

Keywords hospitalized patients; interprofessional collaboration; interprofessional team; patient perspective; patient safety

JBI Database System Rev Implement Rep 2017; 15(8):2020-2027.

\section{Background}

A ccording to the landmark Institute of Medicine reported "to err is human", health care systems are unsafe, with an estimated 44,000 to 98,000 deaths each year in hospital settings, due to medical errors. ${ }^{1} \mathrm{~A}$ recent report revealed that those numbers might be far underestimated. ${ }^{2}$ The Swiss Scientific Advisory Board reported that $10 \%$ of hospitalized patients have been aware of errors committed during their care trajectory. ${ }^{3} \mathrm{Up}$ to $11 \%$ of these patients were particularly concerned with poor care coordination. A majority of errors across all types of industries are due to communication failures. ${ }^{4}$ In the healthcare setting, up to $70 \%$ of errors leading to adverse events (AE) are due to communication breakdown, ineffective communication, and disruptive behavior between nurses and physicians. ${ }^{5}$ Adverse events do not only harm patients physically, but have an influence on patients' mental well-being leading to psychological distress, with feelings of

Correspondence: Amélia Didier, amelia.didier@hesav.ch

There is no conflict of interest in this project.

DOI: 10.11124/JBISRIR-2016-003302 fear, being threatened, and mistrust towards professionals. ${ }^{6}$ Patients consider a lack of interprofessional collaboration (IPC) to be a factor for ineffective care in community settings. ${ }^{7}$ A systematic review on the patient's perspective of $\mathrm{AE}$ in hospitals reported that patients felt generally safe. ${ }^{6}$ Nevertheless, $40 \%$ to $50 \%$ of patients had concerns about safety at some point during hospitalization and reported high rates $(2.9 \%$ to $16.6 \%)$ of $\mathrm{AE}$. This systematic review highlighted that most of the patients identified poor team coordination as the main cause of $\mathrm{AE} .{ }^{6}$ Considering the importance of IPC, for both patients and professionals, governments are encouraging more effective IPC by promoting the implementation of interprofessional education. ${ }^{8}$

Interprofessional collaboration is recognized as a complex process. ${ }^{9-12}$ Effective interprofessional working relationships are developed and maintained with different parties of the healthcare system such as health professionals, patients, families and communities through the IPC process. ${ }^{13}$ The goal is "to enable optimal health outcomes". ${ }^{13(\mathrm{p} .8)}$ Coordination and collaboration between healthcare professionals, especially between nurses and physicians, are key for improving patient outcomes and ensuring patient 
safety, i.e. preventing injuries due to healthcarerelated procedures. ${ }^{1,14}$ Patient safety varies according to individual professional practice, which depends on communication among and the type of support provided by health professionals to each other. ${ }^{15}$

A large body of knowledge already exists about IPC. Numerous authors have delimited core concepts such as trust, mutual respect, interprofessional competencies, shared goals, power relationships, shared leadership and governance and teamwork. ${ }^{9,16}$ Others have conducted a concept analysis of IPC, ${ }^{11,12}$ or developed models and theories of IPC. ${ }^{10,17-19}$ Despite this growing body of knowledge, implementing these concepts successfully in the clinical setting still remains complex. This complexity is partly due to power differences between health professionals, ${ }^{20}$ divergent comprehensions of IPC, or different backgrounds and professional interests. ${ }^{21}$ A meta-analysis of 51 surveys found that nurses called for more IPC with their medical colleagues, whereas physicians rated existing relationships with nurses as collaborative and satisfying. ${ }^{21}$

Existing systematic reviews on IPC have included two types of studies, those focused on professionals ${ }^{22,23}$ and those centred on patient outcomes, ${ }^{14,24}$ in domains such as cancer, ${ }^{25,26}$ pain, ${ }^{27}$ mental health ${ }^{28}$ and end of life. ${ }^{29}$ Some of these systematic reviews presented overall encouraging evidence of the positive influence of IPC on quality of life, ${ }^{26}$ patient assessment and management, better comprehension of the patients' condition, improved care delivery ${ }^{29}$ and reduced mortality. ${ }^{24}$ Other systematic reviews provided little significant evidence for the positive impact of IPC on patient outcomes. ${ }^{14,25}$ In addition, the existing theories and models on IPC provide insufficient insight into its process. A scoping review of ethnographic studies on IPC reported that a lack of understanding exists on how collaborative protocols truly fail or succeed. ${ }^{30}$ Some frameworks of IPC view patients as partners in the interprofessional care team. ${ }^{13,31,32}$ The integration of the patients' perspective on the comprehension and development of IPC is missing in literature. The majority of existing works on definitions, conceptual basis and outcomes of IPC rely on the perspective of health professionals and researchers. Only one systematic review considered the point of view of non-cancer patients on provision of primary care and IPC in a community setting. ${ }^{7}$ This review emphasized lack of care coordination and lack of communication between healthcare professionals. It remained unclear whether patients, families or health professionals had indicated these gaps. The patient's perspective has primarily been studied in relation to shared decision-making processes, ${ }^{33-35}$ which constitutes one of the elements of IPC. Shared decision-making processes are important regarding $\mathrm{AE}^{6}$ which constitutes one of the main patient outcomes of IPC. A paucity of studies also exists in the pediatric context. ${ }^{36,37}$ Children and adults both can contribute to the understanding of social realities.

Health care systems are moving from a paternalistic to a patient-centred approach. ${ }^{38}$ Patients are perceived to play a key role in the redesigning of health care systems. ${ }^{39}$ Indeed, a new health care paradigm assigns patients to be active partners. ${ }^{38}$ This new person-centred role of the patient is expressed in concepts such as empowerment, ${ }^{40}$ patient participation, and patient involvement. ${ }^{38,41}$ However, how do patients themselves perceive their own roles within the health care team and in the care process? How do patients view IPC and their role within it? In view of the high rate of reported $\mathrm{AE}$, their consequences on patients, the critical role of interpersonal relationships between health care professionals in the prevalence and incidence of $\mathrm{AE}$ as well as patients' safety during hospitalization, ${ }^{6}$ it is essential to gain a global view of the patient's perceptions and expectations of IPC.

A concept relevant for practice needs to include the patient's perspective. ${ }^{42}$ Some authors maintain that the patient's perspective should be a key component of any healthcare quality improvement strategy. ${ }^{43}$ Patients are more than merely care recipients; they are at the center of the care process and are aware of errors, and the effects of relational and collaborative processes between healthcare professionals. ${ }^{7}$ Moreover, IPC affects the way patients understand information and are satisfied with care. ${ }^{29}$

Patients can contribute to raise the health professional's awareness about the consequences of their actions. ${ }^{31}$ Patients have the potential to observe professionals during the care process and are aware of interpersonal processes between professionals. ${ }^{44}$ Patients can facilitate changes in the behavior of healthcare professionals. ${ }^{45}$ Patients make professionals aware that their actions bear real consequences for individuals. ${ }^{31}$ As such, patients may help professionals to overcome their barriers 
regarding IPC in hospital settings, thus reducing disruptive behaviors. Health professionals will be better able to tailor their interventions to provide optimal health care to patients.

A preliminary search was conducted in the JBI Database of Systematic Reviews and Implementation Reports, the Cochrane Database of Systematic Review, PubMed and CINAHL, and no review (published or in progress) on this topic is currently available.

In the proposed systematic review, the perspectives of patients (adults and children) during their hospital stay will be explored for gaining a better understanding of IPC, its influence on care, safety and well-being, and the role of patients in the IPC process.

\section{Inclusion criteria}

\section{Types of participants}

This review will consider studies that include men and women, adult and pediatric (six to 18 years) hospitalized patients, notwithstanding their diagnoses.

\section{Phenomena of interest}

The phenomena of interest will be patients' perceptions of IPC. This review will consider studies that explore the perspective of patients hospitalized in adult and pediatric ward on IPC, its influence on their care, safety and well-being, and the role of patients in the IPC process.

\section{Context}

This review will consider studies conducted in any cultural or geographical context, including patients hospitalized in an adult or pediatric ward.

\section{Types of studies}

Studies focusing on qualitative data including, but not limited to, methodologies such as phenomenology, grounded theory, ethnography, action research and feminist research will be considered.

\section{Search strategy}

The search strategy aims to find both published and unpublished studies. A three-step search strategy will be utilized in this review. An initial limited search of PubMed and CINAHL will be undertaken followed by an analysis of the text words contained in the title and abstract, and of the index terms used to describe article. A second search using all identified keywords and index terms will then be undertaken across all included databases. Thirdly, the reference list of all identified reports and articles will be searched for additional studies. Studies published in or translated into English, French and German will be considered for inclusion in this review. Studies published from the inception of the database searched through the current date of the review will be considered for inclusion in this review, in order to provide as broad a search as possible

The databases to be searched include: MEDLINE OvidSp, CINAHL, Excerpta Medica Database (Embase), Cochrane Database of Systematic Reviews, Joanna Briggs Institute EBP Ovid SP, Web of science, PsycInfo, Prospero, Sociological Abstracts.

The search for unpublished studies will include: ProQuest Dissertations and Theses, DART-Europe E-theses Portal, The New York Academy of Medicine Grey Literature Collection.

Initial keywords to be used will be: interprofessional collaboration, interprofessional team, multidisciplinary care team, teamwork, patient care team, health personnel, experience, perception,

\section{Assessment of methodological quality}

Qualitative papers selected for retrieval will be assessed by two independent reviewers for methodological validity prior to inclusion in the review using standardized critical appraisal instruments from the Joanna Briggs Institute Qualitative Assessment and Review Instrument (JBI-QARI) (Appendix I). Any disagreements that arise between the reviewers will be resolved through discussion, or with a third reviewer. Authors of primary studies will be contacted for clarification or missing information.

\section{Data extraction}

Qualitative data will be extracted from papers included in the review using the standardized data extraction tool from JBI-QARI (Appendix II). The data extracted will include specific details about the phenomena of interest, populations, study methods, setting, context and findings significance to the review question and specific objectives. Authors of primary studies will be contacted for clarification or missing information. 


\section{Data synthesis}

Data synthesis of the textual data from qualitative research will include three steps; identifying findings, grouping findings and grouping categories into synthesized findings with the JBI-QARI metaaggregative approach, regardless the study methodology. The latter is possible, since the QARI metasynthesis is restricted to the combination of the findings, and not the primary data.

More specifically, the findings will be pooled using JBI-QARI. This will involve the aggregation or synthesis of findings to generate a set of statements that represent that aggregation, through assembling the findings (Level 1 findings) rated according to their quality, and categorizing findings on the basis of similarity in meaning (Level 2 findings). The categories will be subjected to a meta-synthesis to produce a single comprehensive set of synthesized findings (Level 3 findings) that can be used as a basis for evidencebased practice. Where textual pooling is not possible, the data will be presented in narrative form.

\section{Acknowledgements}

The authors would like to thank the co-investigators of the parent study, i.e. Professor Dr. Brigitte Liebig and Professor Dr. med. Jean-Pierre Pfammatter, as well as the scientific collaborator, Mrs Vanessa Alvarado, for their support. The authors would also like to thank Mrs. Béatrice Perrenoud and Professor Cédric Mabire for their support in the development of the protocol.

\section{References}

1. Kohn LT, Corrigan J, Donaldson MS. Institute of Medicine (U.S.), Committee on Quality of Health Care in America. To err is human: building a safer health system. Washington, D.C: National Academy Press; 1999.

2. James JT. A new, evidence-based estimate of patient harms associated with hospital care. J Patient Saf 2013;9(3):122-8.

3. Vincent C. Improving the quality and safety of healthcare in Switzerland: reflections on the federal strategy [Internet]. Federal Office of Public Health; 2013 [cited 2016 Jan 17]. Available from: http://www.swiss-q.admin.ch/pdf/FinalInput-Paper-Feb-14.pdf.

4. Pronovost P, Berenholtz S, Dorman T, Lipsett PA, Simmonds T, Haraden C. Improving communication in the ICU using daily goals. J Crit Care 2003;18(2):71-5.

5. Manojlovich M. Nurse/physician communication through a sensemaking lens: shifting the paradigm to improve patient safety. Med Care 2010;48(11):941-6.
6. Guijarro PM, Aranaz A, Mira JJ, Perdiguero E, Aibar C. Adverse events in hospitals: the patient's point of view. Qual Saf Health Care 2010;19(2):144-7.

7. Oishi A, Murtagh FE. The challenges of uncertainty and interprofessional collaboration in palliative care for noncancer patients in the community: A systematic review of views from patients, carers and health-care professionals. Palliat Med 2014;28(9):1081-98.

8. Hammick M, Freeth D, Koppel I, Reeves S, Barr H. A best evidence systematic review of interprofessional education: BEME Guide no. 9. Med Teach 2007;29(8): 735-51.

9. D'Amour D, Ferrada-Videla M, San Martin Rodriguez L, Beaulieu M-D. The conceptual basis for interprofessional collaboration: core concepts and theoretical frameworks. J Interprof Care 2005;19(S1):116-31.

10. D’Amour D, Goulet L, Labadie J-F, Martín-Rodriguez LS, Pineault R. A model and typology of collaboration between professionals in healthcare organizations. BMC Health Serv Res 2008;8(1):188.

11. Henneman EA, Lee JL, Cohen Jl. Collaboration: a concept analysis. J Adv Nurs 1995;21(1):103-9.

12. Petri L. Concept Analysis of Interdisciplinary Collaboration. Nurs Forum (Auckl) 2010;45(2):73-82.

13. CIHC. Référentiel national de compétences en matière d'interprofessionalisme [Internet]. 2010 [cited 2015 Dec 21]. Available from: http://www.cihc.ca/files/CIHC_IPCompetencies-FrR_Sep710.pdf.

14. Zwarenstein M, Goldman J, Reeves S. Interprofessional collaboration: effects of practice-based interventions on professional practice and healthcare outcomes. Cochrane Database Syst Rev 2009: CD000072.

15. Vincent C. The Essentials of Patient Safety [Internet]. Wiley-Blackwell; 2012 [cited 2015 Dec 28]. Available from: http://www.chfg.org/wp-content/uploads/2012/03/VincentEssentials-of-Patient-Safety-2012.pdf. In press.

16. San Martín-Rodríguez L, Beaulieu M-D, D'Amour D, FerradaVidela $M$. The determinants of successful collaboration: a review of theoretical and empirical studies. J Interprof Care 2005;19(sup1):132-47.

17. Miller S. Midwives' and physicians' experiences in collaborative practice: a qualitative study. Womens Health Issues 1997;7(5):301-8.

18. Corser WD. A conceptual model of collaborative nursephysician interactions: The management of traditional influences and personal tendencies. Res Theory Nurs Pract 1998;12(4):325-41.

19. Sicotte C, D'Amour D, Moreault M-P. Interdisciplinary collaboration within Quebec community health care centres. Soc Sci Med 2002;55(6):991-1003.

20. Price $S$, Doucet $S$, Hall LM. The historical social positioning of nursing and medicine: Implications for career choice, early socialization and interprofessional collaboration. J Interprof Care 2013;28(2):103-9. 
21. Sollami A, Caricati L, Sarli L. Nurse-physician collaboration: a meta-analytical investigation of survey scores. J Interprof Care 2015;29(3):223-9.

22. McCulloch P, Rathbone J, Catchpole K. Interventions to improve teamwork and communications among healthcare staff. Br J Surg 2011;98(4):469-79.

23. Zangaro GA, Soeken KL. A meta-analysis of studies of nurses' job satisfaction. Res Nurs Health 2007;30(4):445-58.

24. Martin JS, Ummenhofer W, Manser T, Spirig R. Interprofessional collaboration among nurses and physicians: making a difference in patient outcome. Swiss Med Wkly 2010;140:w13062.

25. Pillay B, Wootten AC, Crowe H, Corcoran N, Tran B, Bowden $\mathrm{P}$, et al. The impact of multidisciplinary team meetings on patient assessment, management and outcomes in oncology settings: A systematic review of the literature. Cancer Treat Rev 2016;42:56-72.

26. Gagliardi AR, Dobrow MJ, Wright FC. How can we improve cancer care? A review of interprofessional collaboration models and their use in clinical management. Surg Oncol 2011;20(3):146-54.

27. Ravenek MJ, Hughes ID, Ivanovich N, Tyrer K, Desrochers C, Klinger $L$, et al. A systematic review of multidisciplinary outcomes in the management of chronic low back pain. Work 2010;35(3):349-67.

28. Archer J, Bower P, Gilbody S, Lovell K, Richards D, Gask L, et al. Collaborative care for depression and anxiety problems. Cochrane Database Syst Rev [Internet]. 2012 Oct 17 [cited 2016 Sep 25]; Available from: http://onlinelibrary.wiley.com/doi/10.1002/14651858.CD006525.pub2/Abstract.

29. Puntillo KA, McAdam JL. Communication between physicians and nurses as a target for improving end-of-life care in the intensive care unit: challenges and opportunities for moving forward. Crit Care Med 2006;34(11):S332-40.

30. Paradis E, Leslie M, Puntillo K, Gropper M, Aboumatar HJ, Kitto $S$, et al. Delivering Interprofessional Care in Intensive Care: A Scoping Review of Ethnographic Studies. Am J Crit Care 2014;23(3):230-8.

31. Howe A. Can the patient be on our team? An operational approach to patient involvement in interprofessional approaches to safe care. J Interprof Care 2006;20(5):527-34.

32. Jeffs L, Dhalla I, Cardoso R, Bell CM. The perspectives of patients, family members and healthcare professionals on readmissions: preventable or inevitable? J Interprof Care 2014;28(6):507-12.
33. Kvale $\mathrm{K}$, Bondevik M. What is important for patient centred care? A qualitative study about the perceptions of patients with cancer. Scand J Caring Sci 2008;22(4): 582-9.

34. Mazor KM, Roblin DW, Greene SM, Lemay CA, Firneno CL, Calvi J, et al. Toward patient-centered cancer care: patient perceptions of problematic events, impact, and response. J Clin Oncol 2012;30(15):1784-90.

35. Stewart M, Brown J, Donner A, McWinney IR, Oates J, Weston WW, et al. The impact of patient-centered care on outcomes. Fam Pr 2000;49:796-804.

36. Gazarian M, Graudins LV. Long-term reduction in adverse drug events: an evidence-based improvement model. Pediatrics 2012;129(5):e1334-42.

37. Woods D, Thomas E, Holl J, Altman S, Brennan T. Adverse events and preventable adverse events in children. Pediatrics 2005;115(1):155-60.

38. Thórarinsdóttir K, Kristjánsson K. Patients' perspectives on person-centred participation in health care: A framework analysis. Nurs Ethics 2013;21(2):129-47.

39. Coulter A, Ellins J. Patient-focused interventions: a review of the evidence [Internet]. Health Foundation London; 2006 [cited 2016 Mar 28]. Available from: https://www.researchgate.net/profile/Angela_Coulter/publication/230687402_ The_quality_enhancing_interventions_project_patientfocused_interventions/links/09e415082b83388c9b000000. pdf.

40. Morgan S, Yoder LH. A concept analysis of person-centered care. J Holist Nurs 2012;30(1):6-15.

41. Longtin Y, Sax H, Leape LL, Sheridan SE, Donaldson L, Pittet D. Patient Participation: Current Knowledge and Applicability to Patient Safety. Mayo Clin Proc 2010;85(1): 53-62.

42. Risjord M. Rethinking concept analysis. J Adv Nurs 2009;65(3):684-91.

43. Vincent C, Coulter A. Patient safety: what about the patient? Qual Saf Health Care 2002;11(1):76-80.

44. Henry BW, McCarthy DM, Nannicelli AP, Seivert NP, Vozenilek JA. Patients' views of teamwork in the emergency department offer insights about team performance. Health Expect 2013;19(3):702-15.

45. Mockford C, Staniszewska S, Griffiths F, Herron-Marx S. The impact of patient and public involvement on UK NHS health care: a systematic review. Int J Qual Health Care 2012;24(1):28-38. 
Appendix I: QARI appraisal instrument

\section{JBI QARI Critical Appraisal Checklist for Interpretive \& Critical Research}

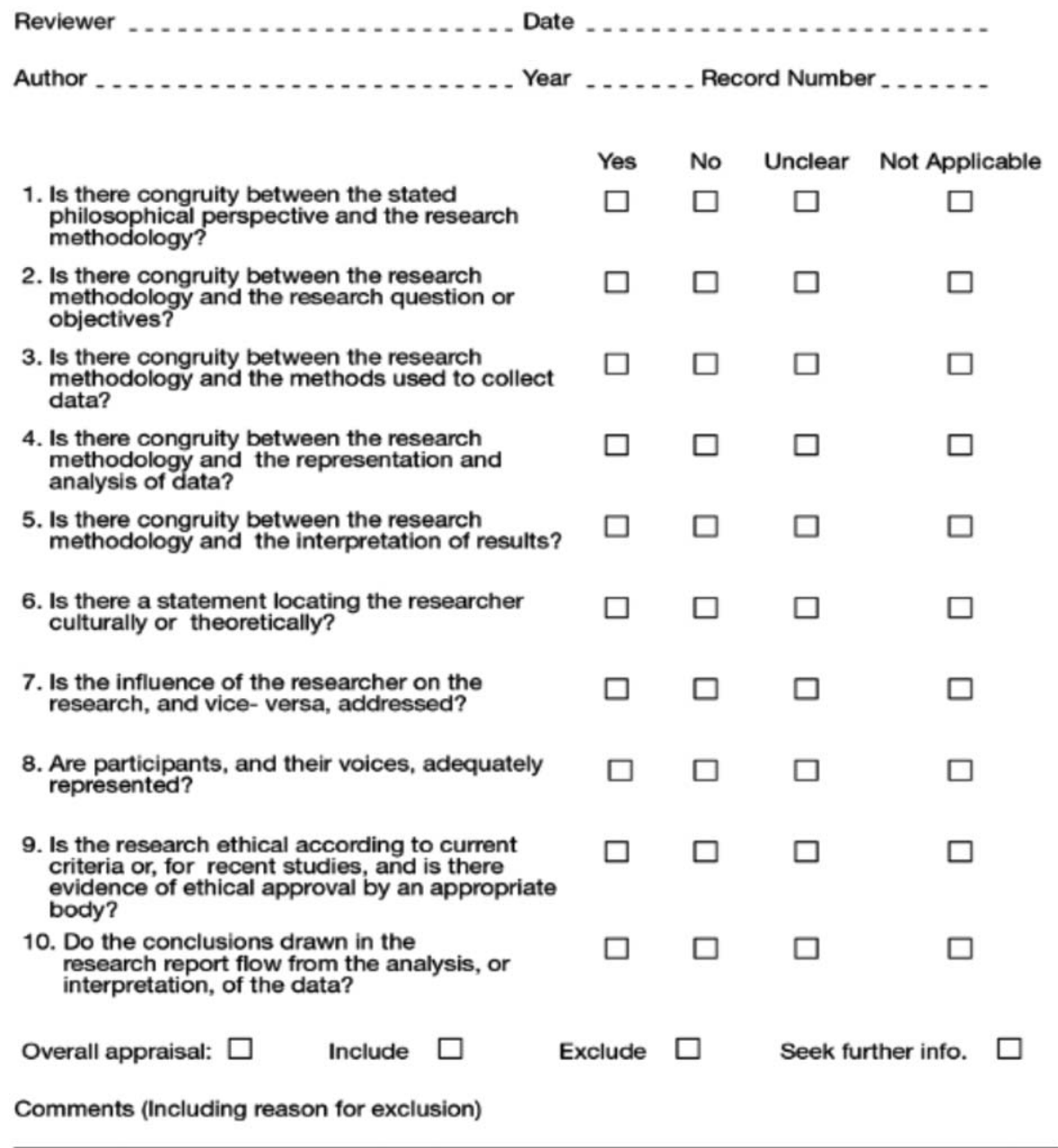


Appendix II: QARI data extraction instrument

\section{JBI QARI Data Extraction Form for Interpretive \& Critical Research}

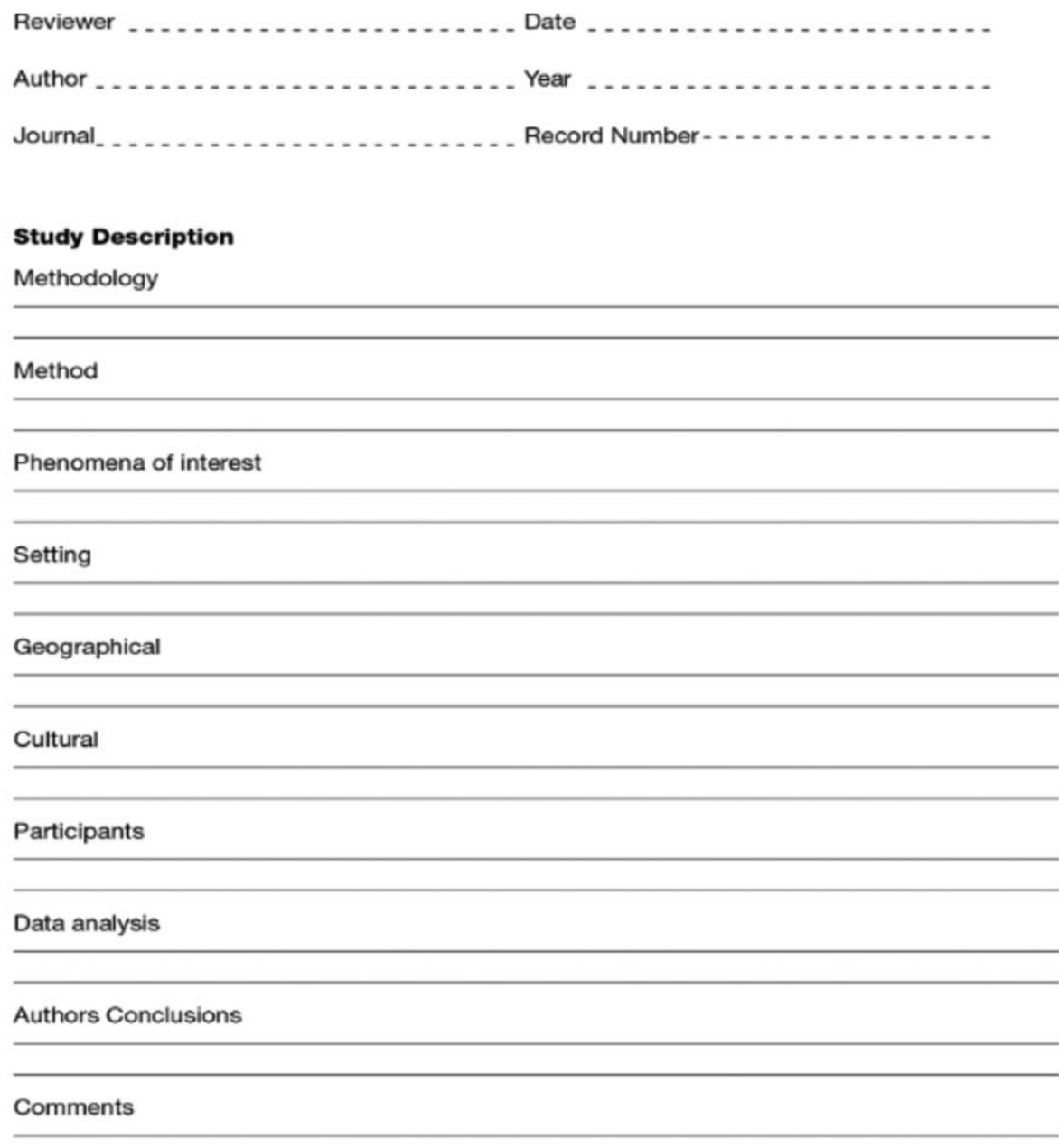

$\begin{array}{lll}\text { Complete } & \text { Yes } \square \quad \text { No } \square\end{array}$ 


\begin{tabular}{|l|l|l|l|l|}
\hline \multirow{2}{*}{ Findings } & \multirow{2}{*}{$\begin{array}{c}\text { Mlustration from } \\
\text { Publication } \\
\text { (page number) }\end{array}$} & Unequivocal & Credible & Unsupported \\
\cline { 3 - 5 } & & & & \\
\hline & & & & \\
\hline & & & & \\
\hline & & & & \\
\hline & & & & \\
\hline & & & & \\
\hline & & & & \\
\hline & & & & \\
\hline & & & & \\
\hline & & & & \\
\hline
\end{tabular}

